\title{
Saline irrigations following sinus surgery - a controlled, single blinded, randomized trial*
}

\author{
Aris I. Giotakis', Eva-Maria Karow², M. O. Scheithauer ${ }^{3}$, Rainer Weber4, \\ Herbert Riechelmann' \\ Rhinology 54: 302-310, 2016 \\ DOI:10.4193/Rhino16.026 \\ ' Department of Otorhinolaryngology, Medical University of Innsbruck, Austria \\ *Received for publication: \\ 2 Siemens and Co, Arzbacher Str. 78, 56130 Bad Ems, Germany \\ January 25, 2016 \\ ${ }^{3}$ Department of Otorhinolaryngology, Head and Neck Surgery, University of Ulm, Germany \\ Accepted: May 17, 2016
}

${ }^{4}$ Department of Otorhinolaryngology, Clinical Centre Karlsruhe, Germany

\begin{abstract}
Background: Chronic rhinosinusitis (CRS) is a common health problem. If medical treatment fails, endonasal sinus surgery is a valuable treatment option. A thorough postsurgical treatment is needed including, among others, nasal saline irrigations (NSI). In this prospective, controlled, single blinded, randomized trial, we aimed to evaluate efficacy of nasal saline irrigations following endonasal sinus surgery in CRS-patients with nasal polyps.
\end{abstract}

Methodology: We examined patient's nasal symptoms, general quality of life and postoperative condition of the mucosa. We also investigated whether or not NSI reduced the number of missed workdays after surgery (MWD). Patients were randomized into an irrigation and non-irrigation arm.

Results: Following treatment, mean nasal sum-score in the irrigation arm was 4.4 and in the non-irrigation arm it was 6.3. Accordingly, mean general sum-score in the irrigation arm was 2.5 and in the non-irrigation arm 4.8. Thus, nasal irrigation led to a more pronounced improvement of nasal and general symptoms than in the non-irrigation arm. No differences were observed in postoperative condition of mucosa or number of MWD.

Conclusions: Nasal irrigation improves symptoms score after ESS in patients with CRSwNP.

Key words: nasal polyps, nasal lavage, controlled clinical trial, quality of life, absenteeism

\section{Introduction}

CRS is estimated to affect $5-10 \%$ of the general population ${ }^{(1,2)}$. Chronic Rhinosinusitis with (CRSwNP) or without nasal polyps (CRSsNP) has a significant socio-economic impact ${ }^{(3)}$. Intranasal and systemic steroids and nasal irrigation are considered first line medical treatment options. Endoscopic sinus surgery (ESS) is considered for patients who fail conservative treatment ${ }^{(3,4)}$. A prolonged postoperative follow-up treatment and observation period may be indicated. Post-ESS treatment mainly includes nasal debridement ${ }^{(5-8)}$ and sprays ${ }^{(9,10)}$. Postoperative nasal debridement is widely used. Repeated nasal debridement does not seem to provide significant symptomatic benefit after ESS (11, 12). Especially in CRSwNP, long-term nasal steroid treatment is often administered as postoperative treatment ${ }^{(10,13)}$.
Multiple data support the positive effect of nasal irrigation as post-ESS treatment ${ }^{(14-16)}$. Differential effects of several solutions for postoperative nasal irrigations have been investigated in prospective clinical trials. Specifically, Low et al investigated the clinical effect of normal saline, lactated Ringer's and hypertonic saline solution on nasal symptoms of 74 patients after ESS and reported that lactated Ringer's results in better improvement compared to normal saline and hypertonic saline solution (17). Faraq et al. compared the clinical effect of surfactant and hypertonic saline on nasal symptoms of 40 patients. The authors concluded that there was no significant difference between the two solutions, however, $20 \%$ of the patients receiving surfactant irrigation stopped the solution due to tolerability issues ${ }^{(18)}$. 
Since blinding is not possible while comparing postoperative nasal irrigations versus a treatment without irrigation, data supporting the efficacy of irrigations as a whole are sparse. Freeman et al., in a randomized controlled trial, investigated the effect of nasal irrigation in the postoperative outcome regarding the presence of adhesions, polyps, crusting, discharge or oedema (19). After applying nasal irrigation at only one side of the nasal cavity, they examined their patients 3 weeks and 3 months following surgery. Postoperative nasal irrigation significantly improved the presence of nasal discharge $(p=0.046)$ and tended to improve mucosal oedema ( $p=0.059$ ). No effect of postoperative nasal irrigation was observed regarding polyps $(p=0.32)$ and adhesions or crusting after 3 weeks and after 3 months.

In this controlled, single blinded, randomized trial, we examined postoperative nasal symptoms, condition of nasal mucosa, quality of life and missed workdays in patients with CRSwNP treated with and without nasal irrigation in a post-ESS follow-up period of 12 months.

\section{Materials and methods}

\section{Study design}

In a prospective, controlled, single blinded, randomized, multicentre trial, patients with CRSwNP scheduled for endonasal sinus surgery were assigned to 2 postoperative treatment arms. Patients in one arm received post-ESS nasal irrigation and patients in the other arm did not. The study was conducted in 6 study centres. Blocked randomization stratified by clinical centre was used to assign patients to the treatment arms. Irrigation treatment included postoperative twice-daily nasal irrigation with $250 \mathrm{ml}$ of 1,175\% Emser Salt ${ }^{\circledR}$ solution (EmsSalt) or an isosmotic mineral salt mixture (IsoMix) resembling ionic composition and concentration of nasal secretions (Siemens \& Co GmbH, Bad Ems, Germany). Emser Salt mainly differs from other salt mixtures by its high sodium bicarbonate content. For irrigation, patients used a flexible plastic bottle with a special tip to fit the nostril. Patients were instructed to let the solution flow through the nostrils while the mouth is kept open. Patients in the non-irrigation arm received standard postoperative nasal care including regular debridement and nasal steroids, but no nasal irrigations.

The trial was approved by the leading institutional review board (ethics committee of the University of Ulm, Germany) and by the ethic committees of each participating centre. Patients were recruited and randomized before the surgical procedure. Surgery was adapted to the extent of disease, but at least uncinectomy, maxillary antrostomy and anterior ethmoidectomy were performed in all patients. Merocel nasal packs were placed bilaterally and were removed at the first or second postoperative day. At the first postoperative clinical examination 3 to 8 days following surgery and following removal of nasal package, base line scores were recorded, patients received their study medication and detailed instructions for their use were given. Clinical follow up visits were scheduled 6 and 12 months after surgery. In addition, patients were contacted 3 and 9 months following surgery by telephone and their symptoms, quality of life, concomitant medication, missed workdays as well as any adverse events were recorded.

\section{Inclusion and exclusion criteria}

Patients between 18 and 60 years of age with CRSwNP according to the definition of the European Academy of Allergy and Clinical Immunology ${ }^{(3)}$ were eligible. Patients with bilateral Malm Grade 1 and $2^{(20)}$ sinonasal polyps who had undergone ESS within the last 8 days were consecutively included. Patients who suffered from a CRSwNP Malm Grade 3 on one side, with a LundMackay CT score $>16^{(21)}$, with documented aspirin exacerbated respiratory disease, women in pregnancy or in lactation period and otherwise severely ill patients were excluded.

Treatment

The patients in the irrigation group were obliged to use the Emser Salt or the isosmotic mineral salt mixture (Siemens \& Co, Bad Ems, Germany) twice daily (Table 1). The solutions were prepared by mixing a pouch of nasal salt in $250 \mathrm{ml}$ of water. The Emser Nasal Irrigation ${ }^{\circledR}$ device was then used to apply the mixture in the nasal cavity twice a day. Compliance was checked after calculating the used and non-used test samples. Concomitant medication was recorded in the patient diary. Patients were allowed to use nasal sprays with budesonide in limited doses in the first 6 months and corticoid and antibiotic nasal ointment in the first 14 days. This was also the allowed treatment in the non-irrigation group. Topical treatment of the nasal mucosa with solutions, sprays, aerosols and nasal ointment as well as topical or systemic expectorants were not allowed as concomitant medication in both treatment arms.

\section{Outcome parameters}

Outcome parameters included a sum score of patient's nasal symptoms, a sum score of patients general quality of life, scores for the condition of nasal mucosa as judged by the treating clinician, and the number of missed workdays (MWD) within the first year following surgery. Nasal symptoms were evaluated using disease specific items from the German version of the Juniper Rhinitis Quality of Life Questionnaire ${ }^{(22)}$. The following nasal symptoms were included: stuffy nose, running nose, mucus and crust formation, posterior rhinorrhea, dry nose and epistaxis. The evaluation was performed by using the following scaling of impairment: 0 = none; 1 = barely; 2 = slightly; 3 = moderately; 4 $=$ quite; 5 = very; 6 = extremely. The addition of the points of the single nasal symptoms gave the nasal sum-score. Accordingly, a 
Table 1. Ionic composition of natural Emser Salt and isosmotic mineral salt mixture.

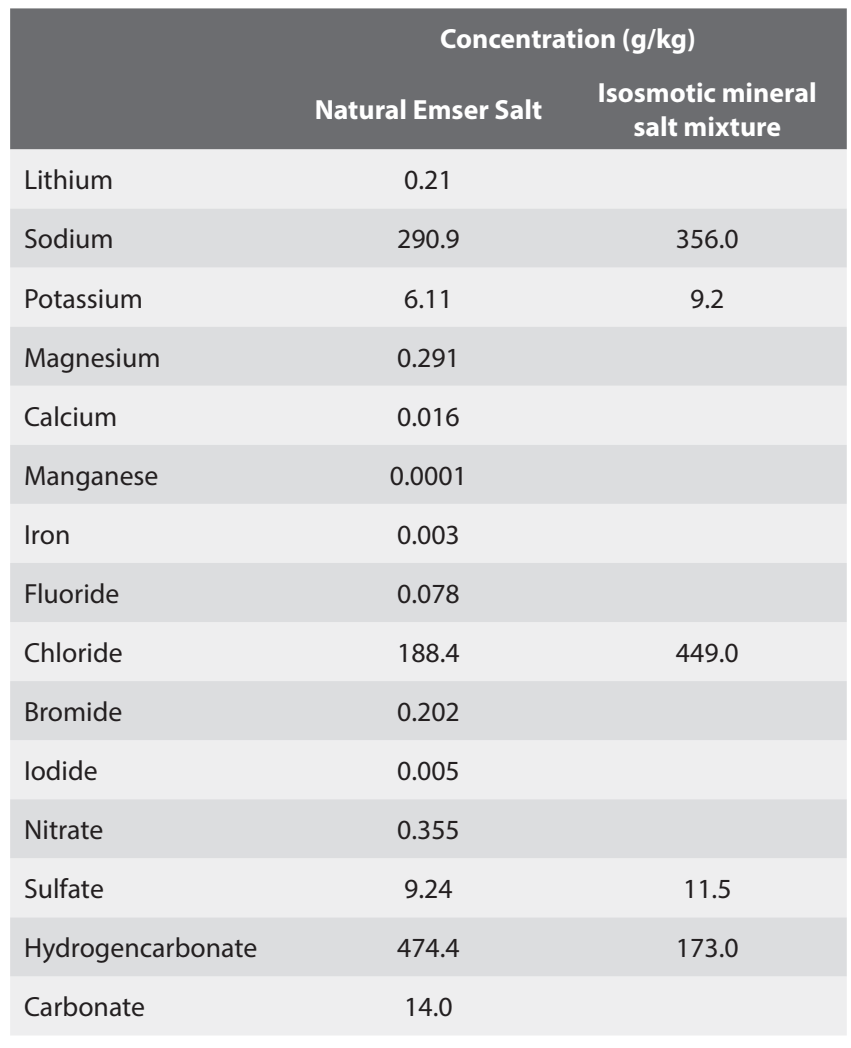

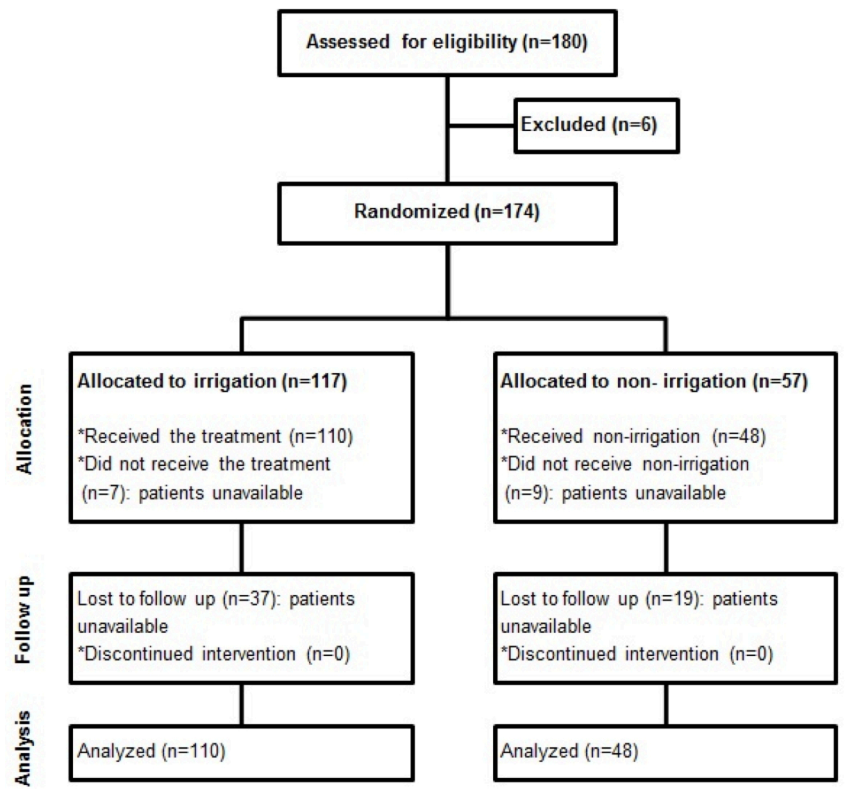

Figure 1. Consort Flow Diagram. Of the 174 patients included, 57 patients were assigned to the non-irrigation arm, 59 patients were assigned to receive Emser Salt and 58 to receive the isosmotic mineral salt mixture. No relevant or significant differences were observed between the Emser Salt group and the Isosmotic mineral salt mixture group (data not shown), and these arms were combined to a common irrigation arm. maximum nasal sum-score of 36 was achievable.

General quality of life was evaluated using disease-unspecific items from the Juniper Rhinitis Quality of Life Questionnaire ${ }^{(23)}$. The scoring principle was the same as in nasal symptom scores. Quality of life items included concentration problems, tiredness, fatigue, general discomfort, sleeping problems, headaches, impairment of professional activities, impairment of leisure activities and impairment of sport activities. The addition of the points of the single general quality of life symptoms gave the general sum-score. Accordingly, a maximum general sum-score of 54 was achievable.

Condition of nasal mucosa was scored by the treating clinicians using several parameters for the worse nasal cavity at the clinical visits 6 and 12 months following surgery. Scores were assigned for nasal polyps ( $0=$ no polyps, $1=$ Malm Grade 1, $2=$ Malm Grade 2 and $3=$ Malm Grade 3), dryness or atrophy ( $0=$ normal mucosa, 1 = dry and slight irritation, 2 = intense atrophy) and scarring $(0=$ no synechiae, $1=$ synechiae between middle turbinate and lateral nasal wall, 2 = extreme lateralization of middle turbinate). These parameters were graded by the investigators using nasal endoscopy.

To document missed workdays, patients were asked in quarterly intervals for the recent quarter. This was done at each regular visit and at 3 and 9 months following start of treatment by telephone interview. At the end of trial, the MWD certificates were collected and evaluated.

Patients were specifically asked for adverse drug effects. If present, they were documented by the treating clinicians in every study centre in the appropriate form.

\section{Data analysis}

An intention to treat analysis was carried out. Data for the two irrigation solutions were combined (irrigation arm) and compared with data of the non-irrigation arm. Frequency data was tabulated. For continuous data, means and standard deviations were provided. Pre-treatment, i.e. within 8 days following ESS, nasal sum-scores and general sum-scores were compared with t-tests to assess for initial homogeneity of groups. Missed workdays in both groups were compared with the Mann-Whitney U-test. To compare nasal sum-scores and general sum-scores in the irrigation and non-irrigation arm at the 4 time points following start of treatment, a linear mixed model was chosen. Treatment (irrigation vs. no irrigation) was modelled as a fixed effect and subjects as random effect. Unstructured was used as covariance structure. LSD-adjusted estimated marginal means served as outcome parameters. 


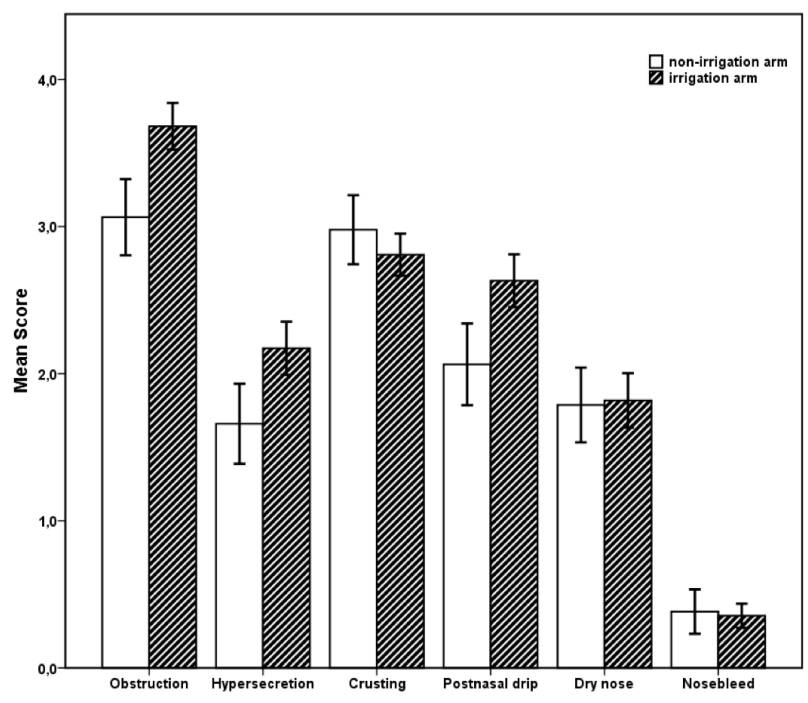

Figure 2. Bar chart representing single nasal symptom scores 1 week following endonasal sinus surgery before start of treatment. The maximum achievable score for a single item was 6 . White bars represent the scores of the non-irrigation arm, shaded bars of the irrigation arm. Error bars represent 1 standard error of mean nasal sum-scores following ESS did not differ between the two treatment arms. Nasal sum scores at start of treatment between both treatment arms did not differ ( $p>0.2)$.

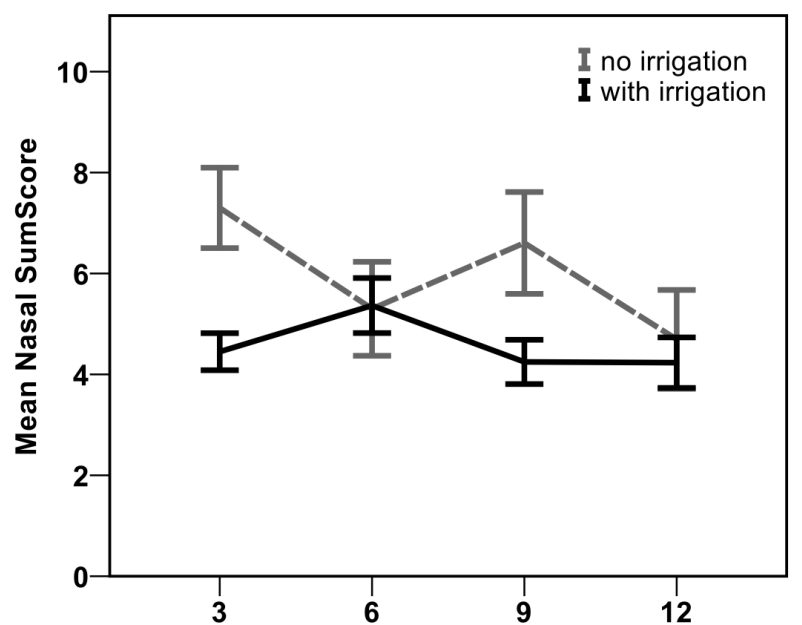

Figure 3. Line chart representing mean nasal sum-scores at the 4 followup times; $y$-axis: mean nasal sum-score (maximum 36 points); $x$-axis: months following start of treatment after endonasal sinus surgery. Error bars represent 1 standard error of mean. The difference between irrigation arm (black solid line) and non-irrigation arm (grey dashed line) was obvious at 3 and 9 months, when participants were interviewed by telephone. Mean nasal sum-score estimated by linear mixed analysis in the irrigation arm at all 4 time points was $4.4(95 \% \mathrm{Cl} 3.8$ to 5.1$)$ and in the non-irrigation arm it was $6.3(95 \% \mathrm{Cl} 5.2$ to $7.3 ; \mathrm{p}<0.01)$. Estimated absolute score difference at all 4 time points was $1.8(95 \% \mathrm{Cl} 0.6$ to 3.1 ; $\mathrm{p}<0.005)$.

\section{Results}

\section{Study population}

Of the 174 patients included, 57 patients were assigned to the control arm and 117 to the irrigation arm. In the irrigation arm, 59 patients were assigned to receive Emser Salt and 58 to receive the isosmotic mineral salt mixture. No significant or clinical relevant differences were observed between the Emser Salt and the isosmotic mineral salt mixture and these arms were combined to a common irrigation arm. A number of 16 patients were excluded from the study because they didn't show up after the initial examination and treatment allocation. Of these, 9 patients belonged to the 57 patients in the control arm and 7 patients belonged to the 117 patients in the irrigation arm. There was a significant difference in the drop-out rate between the two treatment arms (Fisher's exact test; $p<0.05$ ). ITT analysis was performed for 158 patients, of which 110 were in the irrigation arm and 48 in the non-irrigation arm (Figure 1). A number of 56 patients did not complete the study. Among them, 19 were assigned to the non-irrigation arm and 37 to the irrigation arm; no significant difference was observed (Fisher's exact test; $p>0.2$ ). For nasal scores and general QoL-scores, incomplete observations were intrinsically handled by the mixed model analysis. For the number of missed workdays, missing values due to dropouts were compensated carrying the last observation forward.
Mean patient age was 42 years, 103 were men. Results of men and women were well comparable. Mean duration of CRSwNP previous surgery was 6,6 years. Previous ESS had been performed in 43 patients and 2 patients had been operated twice before the current surgical procedure. Perennial allergic rhinitis was diagnosed in 39 patients and seasonal allergic rhinitis in 48 patients. Nasal irrigation could not be concealed from the patients, but the investigators were not aware, if the patients did irrigate or not.

\section{Outcome parameters}

Base-line nasal sum-scores following ESS did not differ between the two treatment arms. They were $13.5 \pm 6.0$ (mean \pm SD, maximum achievable: 36 ) in the irrigation group and $12.1 \pm 7.0$ in the non-irrigation group ( $p>0.2)$. Nasal obstruction, crusting and postnasal drip had the highest scores, whereas nosebleed scored low (Figure 2). Likewise, base-line general sum-scores did not differ between both treatment arms. They were 15.9 \pm 11.9 (maximum achievable: 54 ) in the irrigation arm vs. $13.7 \pm 12.0$ in the non-irrigation arm $(p>0.2)$.

At the first telephone interview following 3 months postoperative treatment, nasal and general sum-scores had improved substantially in both treatment arms. In the irrigation arm, average 


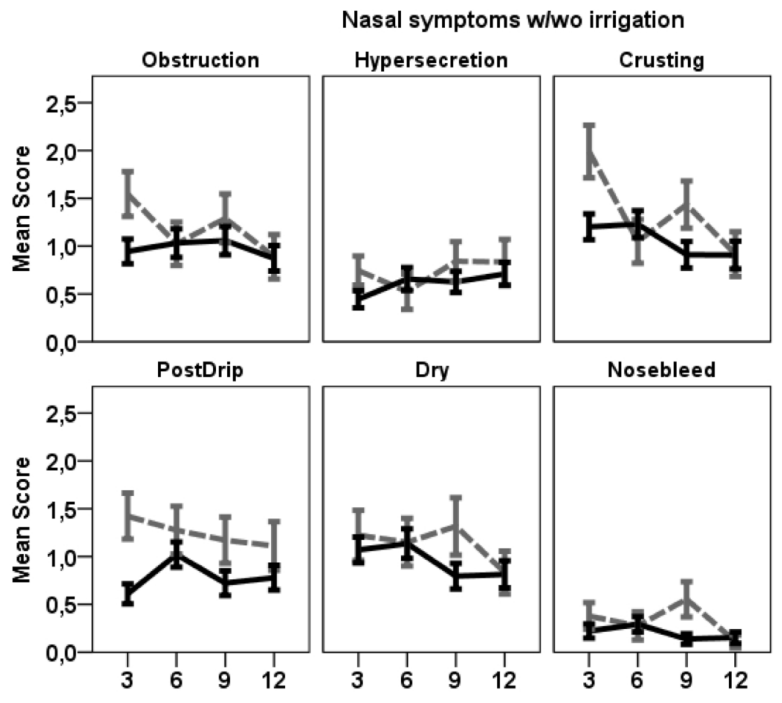

Figure 4 . Single nasal symptom scores at the 4 follow-ups in the irrigation (black solid lines) and non-irrigation arm (grey dashed lines); $x$-axis: nasal symptom score (maximum 6 points); $y$-axis: months following start of treatment. Error bars represent 1 standard error of mean. Visual inspection suggests that nasal irrigation exerted its main effects on postnasal drip, crusting, and mucosal dryness. The differences between the two treatment arms were particularly pronounced at the 1st and 3rd follow up (telephone interviews).
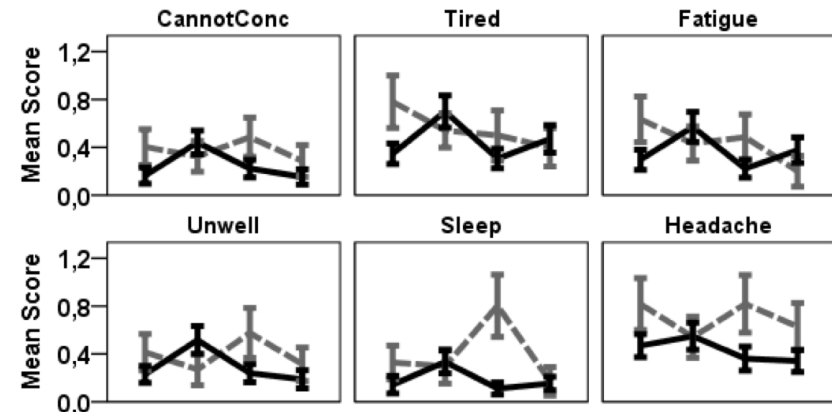

Headache
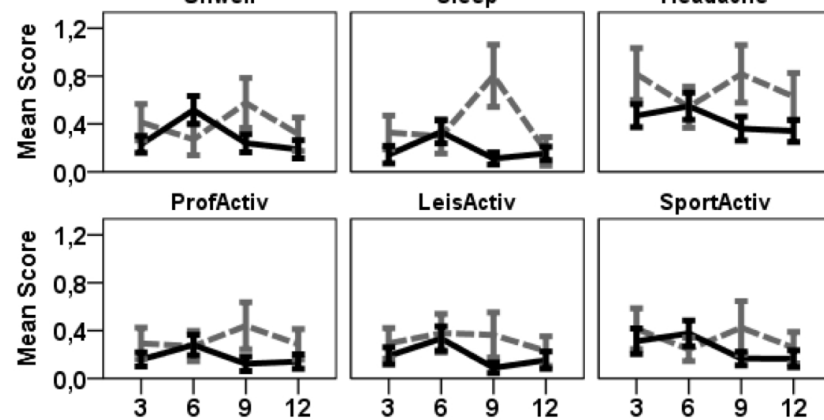

SportActiv

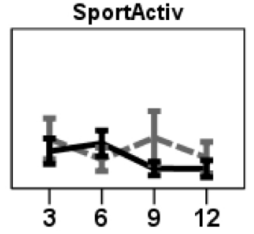

Figure 5. Single general symptom scores at the 4 follow-ups in the irrigation (black solid lines) and non-irrigation arm (grey dashed lines); $x$-axis: general symptom score (maximum 6 points); $y$-axis: months following start of treatment. Error bars represent 1 standard error of mean. Visual inspection suggests that nasal irrigation exerted its main effects on headaches. The differences between the two treatment arms were particularly pronounced at the 1st and 3rd follow up (telephone interviews).

nasal sum-score was $4.5 \pm 3.9$ and in the non-irrigation arm it was 7.3 \pm 5.4. Mean nasal sum-scores remained fairly constant between 4 and 6 score points at the following visits and interview (Figure 3). Estimated marginal means of the linear mixed model provided a single measure to compare both treatment arms accounting for repeated measurements. Estimated mean nasal sum-score in the irrigation arm at the 4 time points was 4.4 (95\% $\mathrm{Cl} 3.8$ to 5.1$)$ and in the non-irrigation arm it was 6.3 (95\% $\mathrm{Cl} 5.2$ to $7.3 ; \mathrm{p}<0.01)$. Breaking the nasal sum-scores down to single symptoms (Figure 4), visual inspection suggests that nasal irrigation exerts its main effects on postnasal drip, crusting, and mucosal dryness. Accordingly, average general sum-score in the irrigation arm had dropped to $2.4 \pm 5.1$ at the first telephone interview and in the non-irrigation arm it had dropped to $5.0 \pm$ 7.1. Mean general sum-score remained fairly constant between 2 and 6 score points at the following visits and interview. Estimated mean general sum-score in the irrigation arm at all 4 time points was 2.5 ( $95 \% \mathrm{Cl} 1.7$ to 3.3 ) and in the non-irrigation arm it was $4.8(95 \% \mathrm{Cl} 3.5$ to $6.1 ; \mathrm{p}<0.01)$. After breaking the general sum-score down to single symptoms (Figure 5), visual inspection suggests that nasal irrigation exerts its main effects on headaches and impairment in sport activities.

During follow up visits 6 months and 12 months after start of treatment, nasal endoscopy was performed and mucosal condition was judged by the treating clinician, who was not aware of the patients treatment allocation. The mean scores of the examined parameters of mucosal condition were similar on day 180 after ESS and day 360 after ESS (Table 2). The change of the mean score of nasal polyps between the day after removal of nasal package and day 360 after ESS was $0.04 \pm 0.7$ (mean score \pm $\mathrm{SD}$ ) in the irrigation arm and $0.08 \pm 0.7$ in the non-irrigation arm. This difference was not statistically significant ( $p>0.2$; table 2 ). For the same period, the change of the mean score of dryness/ atrophy of the nasal mucosa was $0.42 \pm 0.7$ (mean score \pm SD) in the irrigation arm and $0.27 \pm 0.7$ in the non-irrigation arm. This difference was not statistically significant ( $p>0.2)$. Accordingly, the change of the mean score of scarring of the nasal mucosa was $0.01 \pm 0.4$ (mean score \pm SD) in the irrigation arm and $0.03 \pm$ 0.5 in the non-irrigation arm. The difference was not statistically significant ( $p>0.2 ;$ Table 2). The differences of the examined parameters of nasal mucosal condition between the day after removal of nasal package and day 180 after ESS were not statistically significant $(p>0.2)$. The differences of the examined parameters of nasal mucosal condition between day 180 after ESS and day 360 after ESS were not statistically significant ( $p>0.2)$.

The last examined parameter was the number of missed workdays (MWD) in the first postoperative year. In the irrigation arm, the mean number of MWD was $29.5 \pm 37.6$ per patient and in the non-irrigation arm it was $32.8 \pm 53.1 ; p>0.2$. In the first 30 days after ESS, the mean number of MWD in both arms was 13.0 
Table 2. Scores for the condition of nasal mucosa (mean \pm SD) as judged by the examining physicians at nasal endoscopy.

\begin{tabular}{|c|c|c|c|c|}
\hline Parameters & & Irrigation & Non-irrigation & $\mathbf{p}$ \\
\hline \multirow[t]{4}{*}{ Malm Nasal Polyp Grade } & After removal of nasal package & $0.19 \pm 0.5$ & $0.27 \pm 0.6$ & $>0.2$ \\
\hline & Day 180 after ESS & $0.18 \pm 0.3$ & $0.25 \pm 0.5$ & $>0.2$ \\
\hline & Day 360 after ESS & $0.18 \pm 0.4$ & $0.25 \pm 0.6$ & $>0.2$ \\
\hline & $\begin{array}{l}\text { Change of parameter (between removal of nasal } \\
\text { package and day } 360 \text { after ESS) }\end{array}$ & $0.04 \pm 0.7$ & $0.08 \pm 0.7$ & $>0.2$ \\
\hline \multirow[t]{4}{*}{ Dryness/ Atrophy } & After removal of nasal package & $0.55 \pm 0.5$ & $0.46 \pm 0.5$ & $>0.2$ \\
\hline & Day 180 after ESS & $0.21 \pm 0.3$ & $0.29 \pm 0.4$ & $>0.2$ \\
\hline & Day 360 after ESS & $0.19 \pm 0.4$ & $0.29 \pm 0.5$ & $>0.2$ \\
\hline & $\begin{array}{l}\text { Change of parameter (between removal of nasal } \\
\text { package and day } 360 \text { after ESS) }\end{array}$ & $0.42 \pm 0.7$ & $0.27 \pm 0.7$ & $>0.2$ \\
\hline \multirow[t]{4}{*}{ Scarring } & After removal of nasal package & $0.08 \pm 0.3$ & $0.14 \pm 0.3$ & 0.18 \\
\hline & Day 180 after ESS & $0.14 \pm 0.5$ & $0.14 \pm 0.5$ & $>0.2$ \\
\hline & Day 360 after ESS & $0.14 \pm 0.3$ & $0.14 \pm 0.4$ & $>0.2$ \\
\hline & $\begin{array}{l}\text { Change of parameter (between removal of nasal } \\
\text { package and day } 360 \text { after ESS) }\end{array}$ & $0.01 \pm 0.4$ & $0.03 \pm 0.5$ & $>0.2$ \\
\hline
\end{tabular}

per patient. In the remaining 330 days after ESS, the mean number of MWD in the irrigation arm was 16.8 and in the non-irrigation arm it was 19.6. No significant difference between irrigation and non-irrigation arm was noted neither in the first 30 days nor in the remaining 330 days after ESS ( $p>0.2$ ). More than $40 \%$ of the MWD were noted in the first 30 days after ESS.

\section{Adverse events and compliance}

No significant difference in complication rate during and/or after ESS was observed between both treatment arms $(p>0.2)$. Overall, 15 adverse events (AE) were observed in the irrigation arm. Stuffy nose, mucosal oedema, uncomfortable feeling, dry nose and nocturnal rhinorrhoea were observed once, headaches, epistaxis and burning endonasal sensation twice and rhinorrhoea after irrigation four times. During the study, 41 patients (37\%) did not return either used or non-used samples.

\section{Discussion}

Following sinus surgery, tenacious secretions, debris and crusts may accumulate within the nasal cavity due to temporary dysfunction of nasal mucociliary transport. High volume nasal irrigation may support the nasal cleaning process, improve mucosal condition and reduce nasal symptoms during mucosal healing. The effect of different solutions for nasal irrigation has been compared in several studies ${ }^{(17,23)}$. However, there is a paucity of controlled randomized trials comparing postoperative irrigation vs. no irrigation. This might be in part due to inherent problems to conceal the treatment to patients and investigators. Here, we investigated the effect of high volume nasal irrigation in the postoperative care of patients with CRSwNP in a single blinded prospective, controlled, randomized multi-centre trial. Telephone interviewer and physicians doing follow ups were not aware if the patient used nasal irrigations or not. Nasal symptoms, patients' general quality of life, the condition of nasal mucosa and the number of missed workdays (MWD) were evaluated during the first year following endonasal sinus surgery.

In- and exclusion criteria should provide a homogenous group of patients with CRS with nasal polyps. Patients with Malm Grade 3 polyps, with Lund-Mackay score $>16$ and with documented aspirin exacerbated respiratory disease were excluded, because they were considered to suffer from unusually severe disease. In our study population, merocel nasal packing was used. Nasal packing is not obligatory after ESS. Thus, it would be interesting to investigate the effect of nasal irrigation in the absence of nasal packing. In the study protocol, number of missed workdays was the primary outcome parameter. The authors initially investigated whether disease related health scores could be reduced using nasal saline irrigation. An irrigation related difference of the number of missed workdays was not observed ( $p>0.2)$. The analysis of secondary outcome parameters presented here is merely explorative and not confirmative. However, data quality in this prospective trial exceeds that of usual retrospective studies. The data analysis presented here differs in an additional aspect from the study protocol. Initially, patients using irrigation were assigned to two groups receiving different irrigation solutions (Emser Salt and isosmotic mineral salt mixture). Emser Salt contains a higher amount of hydro- 
gencarbonate ions (Table 1). The authors expected that Emser Salt would cause a more neutralized effect in the nasal mucosa. No significant or clinically relevant differences were observed between the Emser Salt and the isosmotic mineral salt mixture arms (data not shown) and these arms were combined to a common irrigation arm.

Base-line nasal symptom scores few days following endonasal sinus surgery and removal of nasal package did not differ in the irrigation and non-irrigation arm (Figure 3). The sum-score reached approximately $40 \%$ of the maximum achievable score of 36 , which is considered surprisingly low so early following removal of nasal package. However, the mean score for nasal obstruction was above $75 \%$ of maximum achievable score in both treatment arms. Nasal obstruction is frequently reported as the most bothersome nasal symptom. Since 'nose bleed' scored low at all time points, sum-scores are generally levelled down. If it is assumed that patients are mainly bothered by the most severe symptoms, sum-scores which include generally low scoring symptoms may underestimate the actual subjective impairment.

At the first follow-up visit 3 months later, sum-scores in both treatment arms had dropped to approximately 5 points, which represents $15 \%$ of the maximum achievable score. This level was similar to the mean level reached by healthy individuals without CRS in 22 Sino Nasal Outcome Test, where mean score was 10.5 (Cl: 9.1 to 11.9) of a maximum achievable score of $67^{(24)}$. At the later follow-up visits, this figure did not change substantially (Figure 3). To compare both treatment arms at the 4 follow up assessments, a linear mixed model for repeated measurements was used ${ }^{(25)}$. At the generally low symptom level of about 5 score points, a significant difference between the two treatment arms was observed. Patients in the irrigation group scored almost 30\% lower than patients in the non-irrigation group. However, the absolute score difference was $1.8(95 \% \mathrm{Cl} 0.6$ to $3.1 ; p<0.005)$ points, which represents only $5 \%$ of the maximum achievable score. The difference between the two treatment arms were particularly pronounced at the first and 3rd follow up (Figure 3), where telephone interviews were performed by one, albeit blinded, interviewer. If the single nasal symptoms items are examined in detail (Figure 4), the most pronounced and constant difference between the irrigation and non-irrigation arms was observed in postnasal drip. Visual inspection was used to avoid multiple testing in a detail-analysis.

Accordingly, base-line general symptom scores few days following endonasal sinus surgery and removal of nasal package did not differ in the irrigation arm and non-irrigation arm. The general sum-score reached approximately $30 \%$ of maximum achievable score of 54 , which is also considered low for this early postoperative period. At the first follow-up visit 3 months following removal of nasal package, general sum-scores in both treatment arms had dropped to approximately 5 points for the non-irrigation arm and 2 points for the irrigation arm. This represents $10 \%$ and $3 \%$ of the maximum achievable score, respectively. This figure remained rather constant in the further follow-ups. At the generally low symptom level of 2-5 score points, a significant difference also of the general sum-scores was observed between the two treatment arms. Patients in the irrigation arm had lower general sum scores than patients in the non-irrigation group. The absolute score difference obtained with a linear mixed model was 2.3 ( $95 \% \mathrm{Cl} 0.8$ to $3.8 ; \mathrm{p}<0.005)$ points, which represents only approximately $4 \%$ of the maximum achievable score. At the single symptom level, differences for headache were most pronounced and fairly constantly observed (Figure 5). Visual inspection was again used to avoid multiple testing in a detail-analysis.

The authors assumed that the patients' health perception mainly depends on the side with worse symptoms. Thus, treating physicians judged the condition of nasal mucosa of the worst nasal cavity within few days following removal of nasal package and at the follow up visits. Scores for nasal mucosa condition were very variable. Standard deviations were many times higher than mean values. Endoscopic judgement of nasal mucosal condition was recently analysed with variable results ${ }^{(26,27)}$. Overall, we did not observe relevant differences between both treatment arms (Table 2). Recurrent nasal polyps and scarring were rarely observed in both treatment arms.

As recently published, CRS resulted in an average of $4.8-5.7$ missed workdays per year in the US ${ }^{(28,29)}$. Bhattacharyya investigated the effect of endoscopic sinus surgery on missed workdays ${ }^{(28)}$ and found a decrease in the mean number of missed workdays from 4.9 to 2.9 per year after ESS ${ }^{(30)}$. In Sweden, CRS and recurrent acute rhinosinusitis (RARS) resulted in an average of $8-14$ missed workdays per year ${ }^{(31)}$. In the present study, the number of missed workdays was $29.5 \pm 37.6$ per patient in the irrigation arm and in the non-irrigation arm it was $32.8 \pm 53.1$; $p>0.2$. In the present study, every missed workday (and not only missed workdays due to sinusitis) was evaluated, because it is basically difficult to differentiate between acute exacerbation of chronic rhinosinusitis or other diseases as cause of missed workdays. Furthermore, missed workdays after ESS were also included; thus explaining the fact the $40 \%$ of missed workdays were observed in the first 30 days after surgery. These could explain the discrepancy in MWD between the present and the other studies ${ }^{(28,29,31)}$. Severe or long lasting adverse events with probable causative relationship to study medication were not observed. A few adverse events were noted, but they were neither severe nor long lasting. Thus, the application of nasal 
irrigation seems to be of low-risk, which has also been verified in earlier studies.

Limitations of this study include the lack of patient concealment. Patients may have heard of and favour postoperative nasal irrigations. Following treatment allocation, more patients allocated to the non-irrigation group did never show up again than patients allocated to the irrigation group $(p<0.05)$. Nasal symptoms were evaluated using disease specific parameters from the German version of the Juniper Rhinitis Quality of Life Questionnaire. The investigators used this questionnaire, because they had previous experience with it from previous clinical trials. However, hyposmia, a frequently presenting symptom in chronic rhinosinusitis with polyps, is not covered in this questionnaire. On the other hand, inclusion of nose bleed, a low scoring symptom, might have levelled down differences between the treatment arms. Moreover, higher scores in the non-irrigation group were recorded in telephone interviews than in clinical follow up visits. No methods, which allow identification of possible investigator bias, had been implemented in the study design. Furthermore, potential differences of nasal mucosa between both groups in an earlier postoperative stage could have been missed. The periodic telephonic and clinical assessment of the patients allowed the first clinical inspection of nasal mucosa 6 months after start of treatment.

\section{Conclusion}

In conclusion, nasal irrigation led to a more pronounced improvement of nasal and general symptoms after ESS and in particular to an improvement of postnasal drip. However, the effect size was low. Moreover, we observed that nasal irrigation had its most pronounced telephonic differences in the telephonic interview in the first 3 months after ESS. Continuing nasal irrigation longer than 3 months after ESS does not seem to provide a relevant benefit.

\section{Acknowledgements}

Participating investigators: M. Scheithauer, MD, (Department of Oto-Rhino-Laryngology, Head and Neck Surgery, Ulm University Medical Center, Germany) W. Heppt, MD (Department of Oto-Rhino-Laryngology, Head and Neck Surgery, Facial Plastic Surgery, Klinikum Karlsruhe, Karlsruhe, Germany). K. Hörmann, MD, (Department of Otorhinolaryngology, Head and Neck Surgery, Mannheim Medical University, Medical Faculty Mannheim of Heidelberg University, Ruprecht-Karls-University, Germany). L. Klimek, MD (Center for Rhinology and Allergology, Wiesbaden, Germany), M. Helbig, MD, (Department of Otorhinolaryngology, Heidelberg University Hospital). R. Jacob, MD, (Bundeswehrzentralkrankenhaus Koblenz, Abt V HNO 56072 Koblenz, Germany).

\section{Authorship contribution}

AIG: Drafting and finalizing the manuscript. HR: Principal investigator, data analysis, finalizing the manuscript; EMK: Project leader, study monitoring, clinical investigator; MOS, RW: Clinical investigators.

\section{Conflict of interest}

The authors declare that there is no conflict of interest.

\section{References}

1. Pilan RR, Pinna FR, Bezerra TF, et al. Prevalence of chronic rhinosinusitis in Sao Paulo. Rhinology. 2012;50(2):129-38.

2. Hastan D, Fokkens WJ, Bachert C, et al Chronic rhinosinusitis in Europe--an underestimated disease. A GA(2)LEN study. Allergy. 2011;66(9):1216-23

3. Fokkens WJ, Lund VJ, Mullol J, et al. EPOS 2012: European position paper on rhinosinusitis and nasal polyps 2012. A summary for otorhinolaryngologists. Rhinology. 2012;50(1):1-12

4. Lund V. Advances in the treatment of nasal polypopsis. Introduction. Allergy. 1999;54 Suppl 53:5-6.

5. Lee JY, Byun JY. Relationship between the frequency of postoperative debridement and patient discomfort, healing period, surgical outcomes, and compliance after endoscopic sinus surgery. Laryngoscope. 2008;118(10):1868-72.

6. Kemppainen T, Seppa J, Tuomilehto H, Kokki $\mathrm{H}$, Nuutinen J. Repeated early debridement does not provide significant symptomatic benefit after ESS. Rhinology. 2008;46(3):23842.
7. Bugten V, Nordgard S, Steinsvag S. Longterm effects of postoperative measures after sinus surgery. Eur Arch Otorhinolaryngol. 2008;265(5):531-7.

8. Nilssen EL, Wardrop P, El-Hakim H, et al. A randomized control trial of post-operative care following endoscopic sinus surgery: debridement versus no debridement. Laryngol Otol. 2002;116(2):108-11.

9. Pinto JM, Elwany S, Baroody FM, Naclerio RM. Effects of saline sprays on symptoms after endoscopic sinus surgery. Am J Rhinol. 2006;20(2):191-6

10. Jang DW, Lachanas VA, Segel J, Kountakis SE. Budesonide nasal irrigations in the postoperative management of chronic rhinosinusitis. Int Forum Allergy Rhinol. 2013;3(9):708-11.

11. Kemppainen T, Seppa J, Tuomilehto H, Kokk $\mathrm{H}$, Nuutinen J. Repeated early debridement does not provide significant symptomatic benefit after ESS. Rhinology. 2008;46(3):23842.

12. Nilssen EL, Wardrop $P, E l$ Hakim $H$, et al. A randomized control trial of post-operative care following endoscopic sinus surgery: debridement versus no debridement. J
Laryngol Otol. 2002:116(2):108-11.

13. Fandino M, Macdonald Kl, Lee J, Witterick IJ. The use of postoperative topical corticosteroids in chronic rhinosinusitis with nasal polyps: a systematic review and meta-analysis. Am J Rhinol Allergy. 2013;27(5):e146-57.

14. Rudmik L, Smith TL. Evidence-based practice: postoperative care in endoscopic sinus surgery. Otolaryngol Clin North Am. 2012;45(5):1019-32

15. Papsin B, McTavish A. Saline nasal irrigation: Its role as an adjunct treatment. CanFamPhysician. 2003;49:168-73.

16. Macdonald KI, Wright ED, Sowerby LJ, et al. Squeeze bottle versus saline spray after endoscopic sinus surgery for chronic rhinosinusitis: a pilot multicentre trial. Am J Rhinol Allergy. 2015;29(1):e13-7.

17. Low TH, Woods CM, Ullah S, Carney AS. A double-blind randomized controlled trial of normal saline, lactated Ringer's, and hypertonic saline nasal irrigation solution after endoscopic sinus surgery. Am J Rhinol Allergy. 2014;28(3):225-31.

18. Farag AA, Deal AM, McKinney KA, et al. Single-blind randomized controlled trial of surfactant vs hypertonic saline irrigation 
following endoscopic endonasal surgery. Int Forum Allergy Rhinol. 2013;3(4):276-80.

19. Freeman SR, Sivayoham ES, Jepson K, de Carpentier J. A preliminary randomised controlled trial evaluating the efficacy of saline douching following endoscopic sinus surgery. Clin Otolaryngol. 2008;33(5):462-5.

20. Malm L. Assessment and staging of nasal polyposis. Acta Otolaryngol. 1997;117(4):465-7.

21. Hopkins C, Browne JP, Slack R, Lund V, Brown P. The Lund-Mackay staging system for chronic rhinosinusitis: how is it used and what does it predict? Otolaryngol Head Neck Surg. 2007;137(4):555-61.

22. Juniper EF, Thompson AK, Ferrie PJ, Roberts $J N$. Validation of the standardized version of the Rhinoconjunctivitis Quality of Life Questionnaire. J Allergy Clin Immunol. 1999;104(2 Pt 1):364-9.

23. Salib RJ, Talpallikar S, Uppal S, Nair SB. A prospective randomised single-blinded clinical trial comparing the efficacy and tolerability of the nasal douching products Sterimar and Sinus Rinse following functional endoscopic sinus surgery. Clin Otolaryngol.
2013;38(4):297-305.

24. Lange B, Thilsing T, Baelum J, Kjeldsen AD. The Sino Nasal Outcome Test 22 score in persons without chronic rhinosinusitis. Clin Otolaryngol. 2015.

25. Norusis MJ. SPSS 14.0 advanced statistical procedures companion. Upper Saddle River, N.J.: Prentice Hall; 2005.

26. McCoul ED, Smith TL, Mace JC, et al Interrater agreement of nasal endoscopy in patients with a prior history of endoscopic sinus surgery. Int Forum Allergy Rhinol. 2012;2(6):453-9.

27. Mace JC, Michael YL, Carlson NE, Litvack JR, Smith TL. Correlations between endoscopy score and quality of life changes after sinus surgery. Arch Otolaryngol Head Neck Surg. 2010;136(4):340-6.

28. Bhattacharyya N. The economic burden and symptom manifestations of chronic rhinosinusitis. Am J Rhinol. 2003;17(1):27-32.

29. Bhattacharyya N. Contemporary assessment of the disease burden of sinusitis. Am J Rhinol Allergy. 2009;23(4):392-5.

30. Bhattacharyya N. Symptom outcomes after endoscopic sinus surgery for chronic rhi- nosinusitis. Arch Otolaryngol Head Neck Surg. 2004;130(3):329-33.

31. Sahlstrand-Johnson P, Ohlsson B, Von Buchwald C, Jannert M, Ahlner-Elmqvist M. A multi-centre study on quality of life and absenteeism in patients with CRS referred for endoscopic surgery. Rhinology. 2011:49(4):420-8.

Aristeidis I. Giotakis, MD, PhD

Department of Otorhinolaryngology

Medical University of Innsbruck

Anichstrasse 35

6020 Innsbruck

Austria

Tel: +43-505-042 3141 